\title{
Pharmacologie moléculaire des neuropeptides
}

La visualisation graphique sur écran d'ordinateur de la forme physique des sites actifs d'enzymes ou de la conformation active de neuropeptides a conduit à la conception logique d'agonistes et d'antagonistes spécifiques. Elle préfigure toute une approche moléculaire nouvelle en pharmacologie.

\section{Bernard P. Roques \\ Professeur à l'université René Des- cartes. Directeur de l'unité U 266 de l'Inserm et de l'unité associée UA 498 du Cnrs.}

\section{REFERENCES \\ I. Roques BP. Enkephalinase inhibitors and molecular exploration of the differences between active sites of enkephalinase and the angiotensin conversion enzyme. I Pharmacol 1985; i6: 5-31. \\ 2. Lord JAH, Waterfield AA, Hughes J, Kos- terlitz HW. Endogenous opioid peptides: multi- ple agonists and receptors. Nature 1977; 265 : 495-9. \\ 3. Roques BP, Garbay-Jaureguiberry C, Oberlin $R$, Anteunis $M$, Lala AK. Conformation of Met ${ }^{5}$-enkephalin determined by high field NMR spectroscopy. Nature 1976; $262:$ 778-9.}

4. Fournie-Zaluski MC, Gacel G, Maigret B, Premilat S, Roques BP. Structural requirements for specific recognition of $\mu$ and $\delta$ opiate receptors. Mol Pharmacol 1981; 20: 484-91.

5. Hansen PE, Morgan BA. Structure-activity relationships in enkephalin peptides. In : Undenfriend S, Meienhofer J, eds. The peptides. New York: Academic Press, 1984: 269-321.

\section{ADRESSE}

B. Roques : département de chimie organique, U 266 Inserm, UA 498 Cnrs, UER des sciences pharmaceutiques et biologiques, 4 , avenue de l'Observatoire, 75270 Paris Cedex o6. e fonctionnement du cerveau exige un transfert d'information qui s'effectue par l'intermédiaire de processus de reconnaissance entre des messagers chimiques et des cibles macromoléculaires chargées de traduire le message. Pour être fonctionnelles ces interactions moléculaires doivent répondre à des exigences de sélectivité liées à la complémentarité des partenaires ou à leur disposition topologique et à des critères de réversibilité nécessaires à la répétitivité du message. La plupart des composants (effecteurs et cibles) des circuits neuronaux ont été identifiés chimiquement au cours de ces dix dernières années et ceci en particulier grâce aux techniques de la génétique moléculaire. Dans certains cas, cette identification s'est étendue au niveau atomique grâce à l'utilisation de méthodes physicochimiques modernes d'analyse structurale (cristallographie aux rayons $\mathrm{X}$, résonance magnétique nucléaire, fluorescence etc.) permettant ainsi une approche rationnelle dans la mise au point de sondes à la fois puissantes et sélectives indispensables pour étudier la physiologie d'une voie neuronale définie. La découverte dans le cerveau d'un grand nombre de neuropeptides a joué un rôle déterminant dans le développement actuel de ces approches rationnelles. Ces neuropeptides constitués pour la plupart d'une succession de 5 à I5 amino-acides sont désormais considérés comme des neurotransmetteurs au même titre que les effecteurs aminergiques (dopamine, adrénaline, acide gamma aminobutyrique etc.) avec lesquels ils peuvent du reste cohabiter dans un même neurone. La biologie des systèmes peptidergiques est cependant plus complexe que celles des monoamines car les neuropeptides sont synthétisés dans le noyau sous forme de précurseurs de haut poids moléculaire dont la maturation s'effectue dans des vésicules de secrétion lors du transport axonal sous l'action d'enzymes dont certaines pourraient être spécifiques. Libérés par exocytose à proximité de récepteurs spécifiques avec lesquels ils interagissent, les neuropeptides sont ensuite métabolisés par des peptidases vraisemblablement impliquées de manière spécifique dans la régulation de la transmission neuropeptidergique. Il est donc théoriquement possible d'étudier en détail le fonctionnement d'un tel circuit à l'aide de molécules capables d'interagir sélectivement 
6. Handa BJ, Lane AC, I ord JAH, Morgan BA, Rance M, Smith CFC.. Analogues of $\beta \mathrm{LPH}_{\mathrm{tl}-\mathrm{N}}$ possessing selective agonist activity at $\mu$-opiate receptors. Eur 7 Pharmacol $1981 ; 70: 531-40$.

7. Zajac JM, Gacel G, Dodey P. et al. Deltakephalin, Tyr-D-Thr-Gly-Phe-Leu-Thr: a new highly potent and fully specific agonist for opiate $\delta$-receptors. Biochem Biophy's Res Commun I983; $111: 390-7$.

8. Mosberg HI, Hurst R, Hruby VJ et al. Bispenicillamine enkephalins possess highly improved specificity toward $\delta$ opioid receptors. Proc Natl Acad Sci USA 1983; 80: 5871-4.

9. Schiller PW. Conformational analysis of opioid peptides and the use of conformational restriction in the design of selective analogs. In : Undenfriend S, Meienhoper I, eds. The peptides. New York: Academic Press, 1985: 220-68.

I0. Dimaio J, Schiller PW. A cyclic enkephalin analog with high in vitro opiate activity. Proc Natl Acad Sci USA i 980; 77: 7162-6.

I I. Maigret B, Premilat S, Fournic-Zaluski MC Roques BP. Proposals for conformation of enkephalins related to opiate $\mathrm{h}$-pharmacophore. Biochem Biophy's Res Commun 1981; 99: 267-74.

12. Burgen ASV, Roberts GCK, Feeney J. Bindings of flexible ligands to macromolecules. Nature 1 975; $253: 753-5$

13. Cotton R, Giles MG, Miller L, Shaw JS, Timms D. ICI 174.864: A highly selective antagonist for the opioid $\delta$-receptor. Eur 7 Pharmacol 1984; $97: 331-2$.

14. Roques BP, Durieux C, Gacel G. et al. Studies on the conformation, enzymatic degradation, pharmacological potency and binding propertics in brain tissue of $\mathrm{CCK}_{8}$ and new related peptides. Ann NY Acad Sci 1985; 448: 6I-75.

15. Chang RSL, Lotti VJ, Monaghan RL, et al. A potent nonpeptide cholecystokinin antagonist selective for peripheral tissues isolated from Aspergillus alliaceus. Science $1985 ; 230: 177-9$.

I6. Quirion R, Zajac JM, Morgat JL, Roques BP. Autoradiographic distribution of $\mu$ and $\delta$ opiate receptors in rat brain using highly selective ligands. Life Sci 1983; 33: 227-30.

17. Chaillet P, Coulaud A, Zajac JM, FournieZaluski MC, Costentin J, Roques BP. The $\mu$ rather than the $\delta$ subtype of opioid receptors appears to be involved in enkephalin-induced analgesia. Eur 7 Pharmacol 1984; 101 : 83-90.

I8. Chesselet MF, Cheramy A, Reisine'TI), et al. Effects of various opiates including specific $\delta$ and $\mu$ agonists on dopamine release from nigrostriatal dopaminergic neurons in vitro in the rat and in vivo in the cat. Life Sci 1982; 31 : 2291-4.

19. Garbay-Jaureguiberry S, Robichon A, Dauge V, Rossignol P, Roques BP. Highly selective photoaffinity labeling of $\mu$ and $\delta$ opioid receptors. Proc Natl Acad Sci USA 1984; 81 : 7718-22. avec une des cibles régulatrices. De telles molécules permettent de définir l'action physiologique du neuropeptide et les modifications biochimiques provoquées par le blocage ou l'inactivation d'un composant du circuit. Ceci s'effectue en mesurant quantitativement non seulement les concentrations du neuropeptide, de ses récepteurs, des enzymes de dégradation, etc., mais également le taux des ARN messagers codant pour ces différentes molécules.

Le meilleur exemple du développement rationnel d'outils nécessaires à l'analyse intégrée du fonctionnement d'un circuit neuropeptidergique est celui des peptides opioïdes enképhalines [I]. La démarche moléculaire empruntée dans la mise au point de ces outils, sera brièvement illustrée dans cet article.

\section{Multiplicité des récepteurs opioides}

La multiplicité des effets pharmacologiques engendrés par l'administration de morphine (analgésie, dépression respiratoire, euphorie, modification du transit intestinal, dépendance physique et psychique etc.) semble due à son action non discriminante au niveau des différents récepteurs. Ceci est en accord avec l'existence dans le cerveau d'une hétérogénéité de sites de liaison pour la morphine mais également pour les peptides morphinomimétiques endogènes. Ainsi la morphine et les enképhalines TyrGly-Gly-Phe-Leu et Tyr-GlyGly-Phe-Met se lient dans le cerveau à deux sites distincts dénommés récepteurs $\mu$ et $\delta[2]$.

Cependant, la morphine manifeste une affinité sensiblement plus grande pour les sites $\mu$ alors que les enképhalines interagissent préférentiellement avec les récepteurs $\delta$. Afin de caractériser les réponses pharmacologiques associées à l'activation sélective de chacun de ces récepteurs, il était indispensable de développer des ligands spécifiques. La démarche moléculaire suivie a consisté à prendre en compte les caractéristiques structurales des agonistes endogènes Leu- et Metenképhaline et de les confronter avec celles des morphiniques synthêtiques. En effet, l'aptitude d'une molécule à se lier à plusieurs récepteurs différents suppose une adaptabilitć conformationnelle lui permettant de satisfaire à des interactions spécifiques dans chacun de ces sites. L'utilisation de la résonance magnétique nucléaire (RMN) [3] a permis de montrer l'existence en solution d'un équilibre entre une forme repliée (avec stabilisation par liaison hydrogène) et une forme plus étendue pour la Met- et la Leu-enképhaline. Des analogies structurales entre le peptide et un dérivé de la morphine (oripavine) possédant un cycle phenyl additionnel ont été mis en évidence. Ainsi, la tyrosine des enképhalines mimerait le reste phényléthyl ammonium présent dans la morphine et ses dérivés. Ce groupe serait indispensable à la reconnaissance de l'un ou l'autre des récepteurs mais la discrimination impliquerait la présence obligatoire d'un reste Phe pour l'interaction avec le récepteur $\delta$ et un raccourcissement couplé à un repliement de la séquence peptidique pour une liaison préférentielle au site $\mu$ [4].

Cette modélisation des pharmacophores $\mu$ et $\delta$ a permis de préparer une séric d'agonistes puissants et sélectifs des deux récepteurs dont les plus intéressants ont été tritiés et sont désormais utilisés comme ligands de référence pour les études biologiques et pharmacologiques [5]. En ce qui concerne les sites $\mu$ les meilleurs agonistes sont le DAGO [6] et le TRIMU 5 ( $f-$ gure $I$, voir $p .2 I$ ) dont la ressemblance avec la morphine est évidemment frappante. Ces composés sont environ $\mathrm{I} 00$ fois plus sélectifs pour les récepteurs $\mu(\mathrm{KD} \sim 2$ à $8 \mathrm{nM})$ que pour les sites $\delta(\mathrm{KD} \sim 200$ à $800 \mathrm{nM}$ ) alors que la morphine montre une affinité io fois plus grande pour les récepteurs $\mu$. Les meilleurs agonistes $\delta$ sont de deux types : linéaires comme le DTLET (Tyr-D-Thr-Gly-Phe-Leu-Thr) [7] et cyclique tel le DPDPE [8] (Tyr-D. Pen-Gly-Phe-D.Pen). Le DTLET a une meilleure affinité $\delta$ (KD I nM) alors que le DPDPE est plus sélectif.

Le modèle d'interaction sélective avec les sites $\mu$ et $\delta$ que nous avions proposé [4] s'est vu conforté par la mise en évidence, également à l'état cristallin, des formes repliée et éten- 


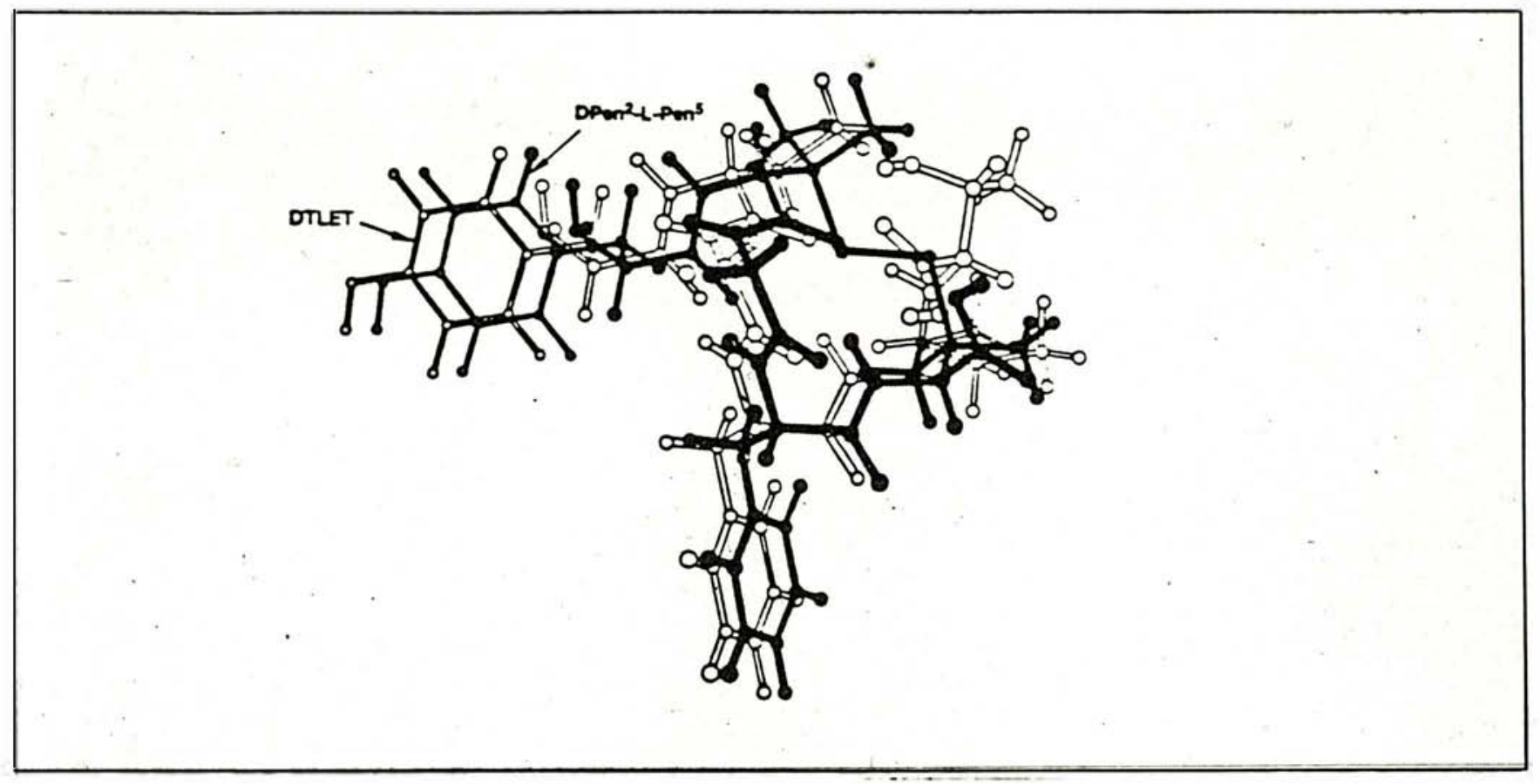

La figure I se trouve en p. $2 I$.

due pour la Leu-enképhaline [9]. Néanmoins, bien que la plupart des peptides cycliques synthétiques manifestent une grande sélectivité $\mu$ [Io], le caractère $\delta$ sélectif du DPDPE semblait contredire le modèle. En fait, les deux composés $\delta$ sélectifs DTLET et DPDPE peuvent adopter des conformations similaires comme l'a récemment montré G. Loew par des calculs de minimisation d'énergie (figure 2). De manière générale, la démarche synthétique suivie a été étayée par des calculs d'énergie conformationnelle qui apportent des informations essentielles sur le caractère réaliste des modèles et surtout sur la déformation subie par la conformation le plus stable en solution lors de la liaison au récepteur [I I]. La confrontation des modèles calculés

Figure 2. Superposition obtenue par ordinateur des enképhalines synthétiques spécifiques du récepteur $\delta$ (G. Loem, (I985) NIDA Research Monograph). L'une est linéaire DTLET, l'autre est cyclique DPDPE (voir texte). Ce type d'analyse permet de calculer l'énergie à fournir pour adapter la conformation de la molécule linéaire à un modèle cyclique dont la structure est supposée relativement proche de celle existant dans le récepteur. L'interaction du neuropeptide avec le récepteur s'effectue par de légères transconformations permettant l'adaptation des chaînes latérales dans les sous-sites de la protéine réceptrice (zipper mechanism).

et des équilibres conformationnels en solution a permis de proposer un modèle de liaison par transconformation adaptative (zipper mechanism [12]) des enképhalines à leur site de liaison. En partant de ces résultats et en bloquant la flexibilité d'une partie de la structure, nous venons de préparer un nouveau ligand $\delta$ linéaire qui garde l'affinité du DTLE.' mais dont la spécificité est supéri ure à celle du DPDPE (G. Gacel et al., résultats non publiés).

Il semble désormais clair que les petits peptides n'interagissent pas avec leurs cibles selon le modèle figé de la clef dans la serrure, et que la traduction du message exige une transconformation du récepteur, probablement concertée avec celle de l'agoniste. Par conséquent, un antagoniste devra satisf aire aux exigences de reconnaissance du site récepteur mais interdire le changement structural permettant la traduction du message qui s'effectuerait, par exemple, par couplage avec une unité enzymatique (adénylate cyclase) ou par ouverture d'un canal. C'est en tenant compte de ces considérations de dynamique moléculaire qu'a été développé un grand nombre d'antagonistes peptidiques tels que le ICI $\mathrm{N}, \mathrm{N}^{\prime}$-diallyl-TyrAib-Aib-Phe-Leu [I3], antagoniste $\delta$ sélectif linéaire dans lequel la rigidité est induite par les restes Aib $\left(\mathrm{H}_{2} \mathrm{~N}-\mathrm{C}\left(\mathrm{CH}_{3}\right)_{2}-\mathrm{COOH}\right)$.

En l'absence de données structurales précises sur les sites actifs des récepteurs (le seul dont la séquence complète est connue est le récepteur cholinergique), la mise au point 


\section{REFERENCES}

20. Cushman DW, Cheung HS, Sabo EF Ondetti MA. Design of potent competitive inhibitors of angiotensin-converting enzyme. Carboxyalkanoyl and mercaptoalkanoyl aminoacids. Biochemistry 1977; 16 : 5484-91

21. Monzingo AF, Matthews BW. Structure of a mercaptanthermolysin complex illustrates mode of inhibition of zinc proteases by substrateanalogues mercaptans. Biochemistry 1982; 21 : 3390-4.

22. Roques BP, Fournie-Zaluski MC, Soroca E et al. The enkephalinase inhibitor thiorphan shows antinociceptive activity in mice. Nature 1980; 288 : 286-8.

23. Roques BP, Lucas-Soroca $E$, Chaillet $P$, Costentin J, Fournie-Zaluski MC. Complete differentiation between enkephalinase and angiotensinconverting-enzyme inhibition by retro-thiorphan Proc Natl Acad Sci USA 1983; 80 : 3178-82.

24. Fournie-Zaluski MC, Chaillet $P$, Bouboutou $\mathrm{R}$ et al. Analgesic effects of kelatorphan, a new highly potent inhibitor of multiple enkephalin degrading enzymes. Eur 7 Pharmacol 1984; 102 : 525-8.

25. Waksman G, Bouboutou R, Devin J et al. In vitro and in vivo effects of kelatorphan on enkephalin metabolism in rodent brain. Eur 7 Pharmacol 1985 , sous presse

26. Petit F, Hamon M, Fournie-Zaluski MC, Roques BP, Glowinski J. $\delta$ opioid receptors in the rat striatum but not in the nucleus accumbens are involved in presynaptic regulation of newly synthesized dopamine release. Eur 7 Pharmacol I985, soumis pour publication.

27. Waksman G, Hamel E, Fournie-Zaluski MC, Roques BP. Comparative distribution of the neutral endopeptidase "enkephalinase " and $\mu$ and $\delta$ opioid receptors in rat brain by autoradiography. Proc Natl Acad Sci USA 1986, sous presse.

28. Lynch DR, Strittmatter SM, Snyder SH. Enkephalin convertase localization by [ ${ }^{3} \mathrm{H}$ ] guanidino ethylmercapotosuccinic acid autoradiography: selective association with enkephalin-containing neurons. Proc Natl acad Sci USA 1984; 81 : 6543-7.

29. Comb M, Seeburg PH, Adelman J, Eiden L, Herbert E. Primary structure of the human Metand Leu-enkephalin precursor and its mRNA. Nature 1982; 295 : 663-6.

30. Yoshikawa K, Hong J, Sabol S. Electroconvulsive shock increases preproenkephalin messenger RNA abundance in rat hypothalamus. Proc Natl Acad Sci USA 1985; 82: 589-93

31. Sutcliffe JG, Milner RJ, Bloom FE, Lerner $\mathrm{RA}$. Common 82-nucleotide sequence unique to brain RNA. Proc Natl Acad Sci USA 1982; 79: 4942-6.

32. Bost KL, Smith EM, Blalock JD. Similarity between the corticotropin (ACTH) receptor and a peptide encoded by an RNA that is complementary to ACTH mRNA. Proc Natl Acad Sci USA $1985 ; 82: 1372-5$

33. Hamel E, Beaudet A. Electron microscopic autoradiographic localization of opioid receptor in rat neostriatum. Nature 1984; 312 : 1 55-7.

34. Denis-Donini S, Glowinski J, Prochiantz A Glial hetcrogeneity may define the threedimensional shape of mouse mesencephalic dopaminergic neurones. Nature 1984; 307 : 641-3. d'agonistes et d'antagonistes bénéficie de l'apport considérable des techniques de visualisation graphique sur écran qui, couplés à des logiciels de calculs d'énergie permettent de modéliser de manière réaliste les changements structuraux induisant la sélectivité.

\section{Modélisation graphique de la cholécystokinine}

Cette approche vient d'être utilisée pour mettre au point des agonistes et antagonistes de la cholécystokinine cérébrale ou $\mathrm{CCK}_{8}$ (Asp-Tyr $\left(\mathrm{SO}_{3} \mathrm{H}\right)-$ Met-Gly-Trp-Met-Asp Phe- $\mathrm{NH}_{2}$ ). Le $\mathrm{CCK}_{8}$ présente un très grand intérêt car localisé avec la dopamine dans certains neurones de la voie mésolimbique, il possèderait un profil proche des neuroleptiques du type halopéridol. Par ailleurs, il se comporte dans de nombreux systèmes (y compris celui du contrôle des influx nociceptifs) comme un antagoniste indirect des peptides opioïdes. Bien qu'ils demandent à être confirmés, ces résultats ont entraîné une intense compétition internationale dans le but d'élucider les propriétés physiologiques du $\mathrm{CCK}_{8}$ et éventuellement d'utiliser ce peptide comme modèle pour synthétiser de nouveaux agents phsychotropes. Le $\mathrm{CCK}_{8}$, comme tous les neuropeptides est dégradé par des peptidases, ce qui nécessite la mise au point d'agonistes et antagonistes résistant à ces enzymes. Ceci peut être obtenu, comme dans le cas des enképhalines, par introduction d'aminoacides modifiés incapables d'être reconnus par les enzymes, ou par synthèse de peptides cycliques incapables d'accéder au site actif des peptidases. Cependant les peptides qui, tel le $\mathrm{CCK}_{8}$, possèdent une tyrosine sulfatée sont d'un accès synthétique difficile, ce qui justifie d'autant mieux l'optimisation des conditions d'accès aux dérivés actifs. Comme pour les enképhalines, nous avons débuté le programme de synthèse par une analyse conformationnelle par RMN qui a montré l'existence de repliements de structure très stables même en solution aqueuse. L'existence des résidus fluorescents $\operatorname{Tyr}\left(\mathrm{SO}_{3} \mathrm{H}\right)$ et Trp a permis en outre de calculer la distance entre ces deux cycles aromatiques par transfert de fluorescence [I4].

La détermination des couplages entre protons donne accès aux angles dièdres qui permettent de définir lá conformation générale du squelette peptidique. La mise en évidence d'effets de relaxation dipolaire internes (effets NOE) qui dépendent de la distance entre les noyaux étudiés $\left({ }^{1} \mathrm{H}\right.$ dans le cas présent) participe à l'affinement d'un modèle conformationnel dit expérimental. Parallèlement, un programme de minimisation d'énergie, tenant compte des attractions et répulsions entre atomes, sélectionne sans a priori (méthode de MonteCarlo) les conformations de plus basse énergie. Dans le cas du $\mathrm{CCK}_{8}$, un million de conformations ont ainsi été triées par l'ordinateur avant de converger vers un petit nombre de structures dont deux sont proches du modèle expérimental (figure 3). Bien entendu, rien n'indiquait que ces conformations étaient identiques aux conformations actives au récepteur mais compte tenu de la taille du peptide, de son caractère amphiphile et de sa très grande affinité, on était en droit de penser que la structure active ne devait pas être trop éloignée du modèle expérimental sous peine d'une pénalisation thermodynamique trop grande lors de la liaison par transconformation adaptative. Partant de ces données nous avons donc préparé le premier dérivé cyclique du $\mathrm{CCK}_{8}$, composé qui possède une affinité voisine de celle de l'effecteur naturel mais dont la stabilité et les propriétés pharmacocinétiques sont supérieures à celles de l'effecteur naturel $\mathrm{CCK}_{8}$. La modification des structures peptidiques par introduction d'aminoacides modifiés ou par cyclisation des structures, montre qu'il est désormais possible de préparer des agonistes et antagonistes répondant à certaines exigences pharmacocinétiques (stabilité, passage de la barrière hématoencéphalique), ce que l'on pensait réservé aux molécules organiques de structure classique. Néanmoins la synthèse de molécules opioïdes appuyée sur les déterminations conformationnelles des effecteurs endogènes (enképhalines) montre que, transposé au cas du $\mathrm{CCK}_{8}$, il 


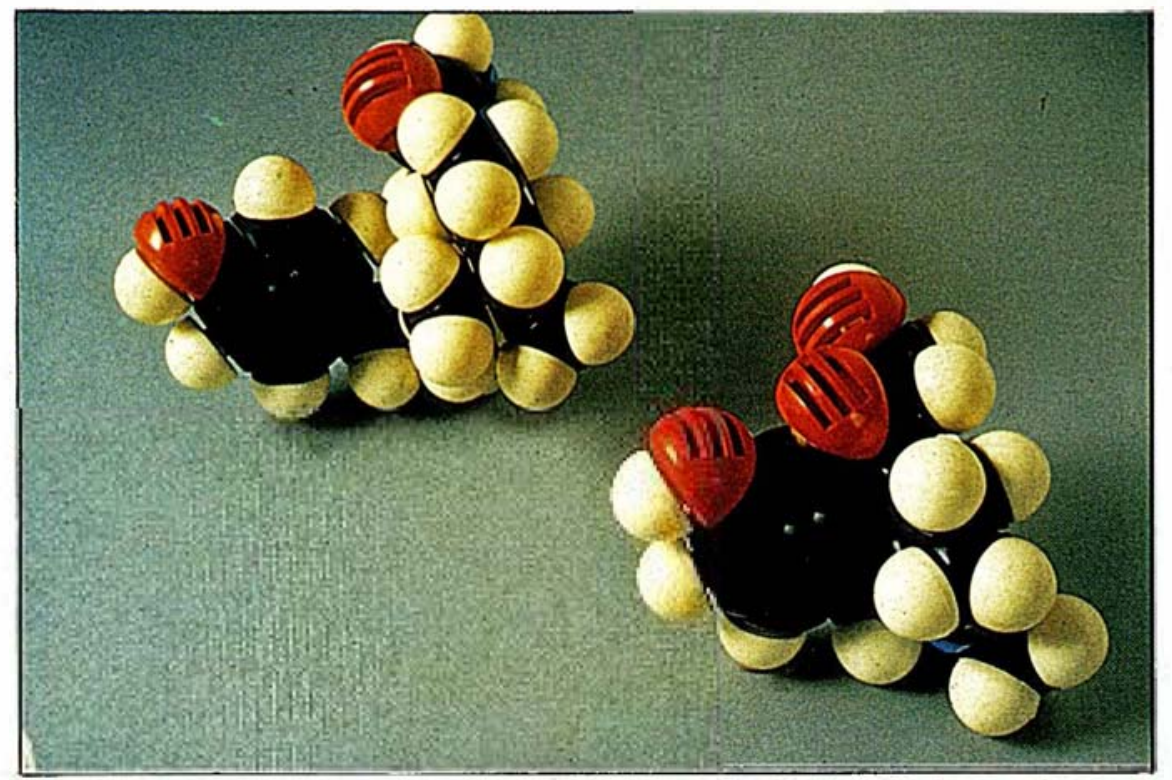

Figure I. Modèles moléculaires de la morphine (droite) et du TRIMU 5 (gauche), un analogue synthétique des enképhalines montrant une grande sélectivité pour les récepteurs $\mu$. On remarque l'analogie dans la disposition spatiale du cycle phénol et du groupe ammonium.

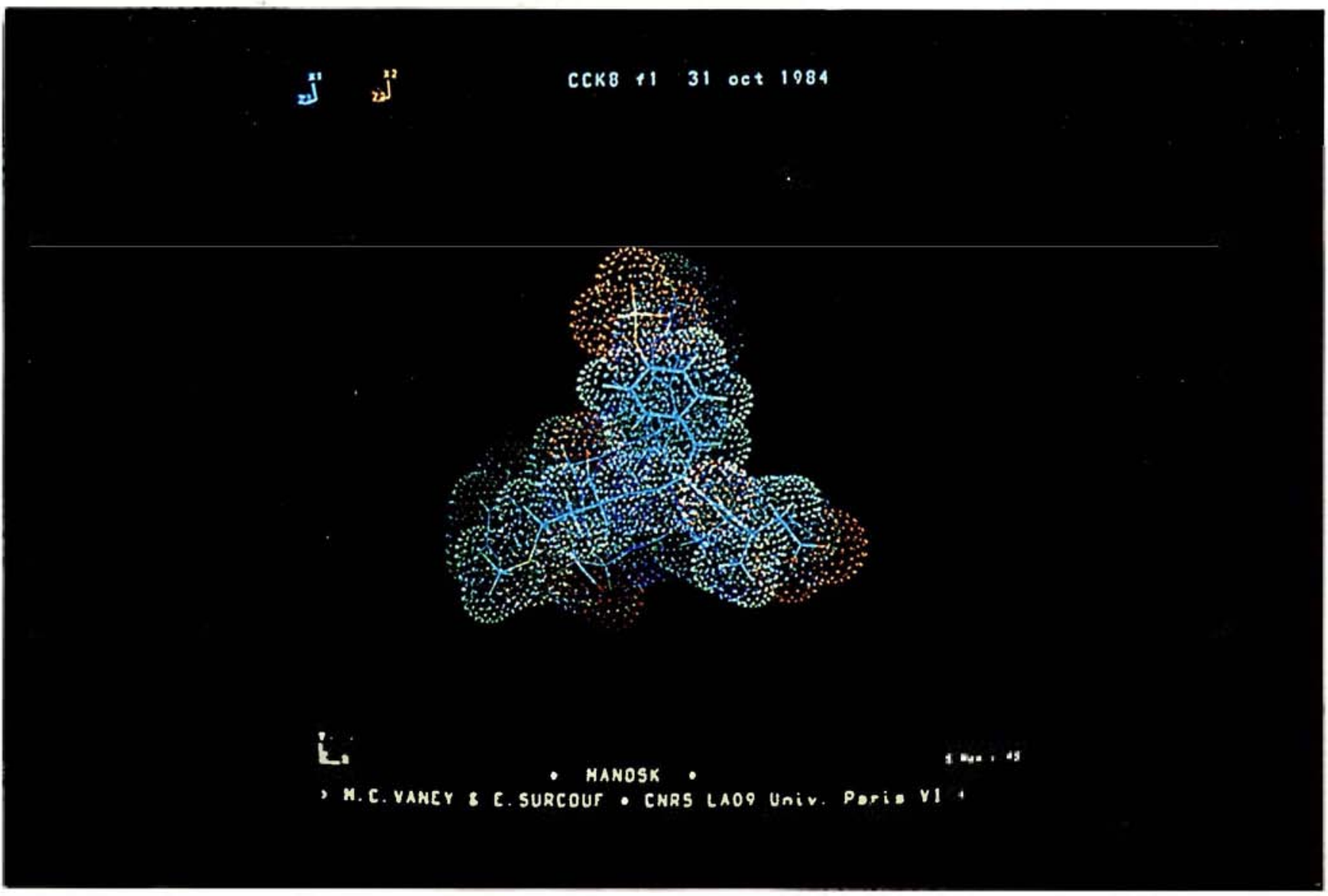

Figure 3. Visualisation sur écran graphique de la conformation du neuropeptide CCK $K_{8}$ déduite d'études par RMN et de calculs conformationnels (coll. B. Maigret, Cnrs, Strasbourg). Des logiciels tels que Manosk, développé par F. P. Mornon et E. Surcouf (Cnrs, Univ. Paris-VI), permettent de modifier la conformation du peptide directement sur l'écran, de calculer l'énergie de chaque conformation, la distance entre les atomes, la superposition possible avec d'autres modèles souples ou rigides etc. Ces techniques représentent donc une aide puissante à la synthèse rationnelle d'agonistes ou antagonistes 'd'un neuropeptide. $m / s n^{\circ}$ I vol. 2 janvier 86 


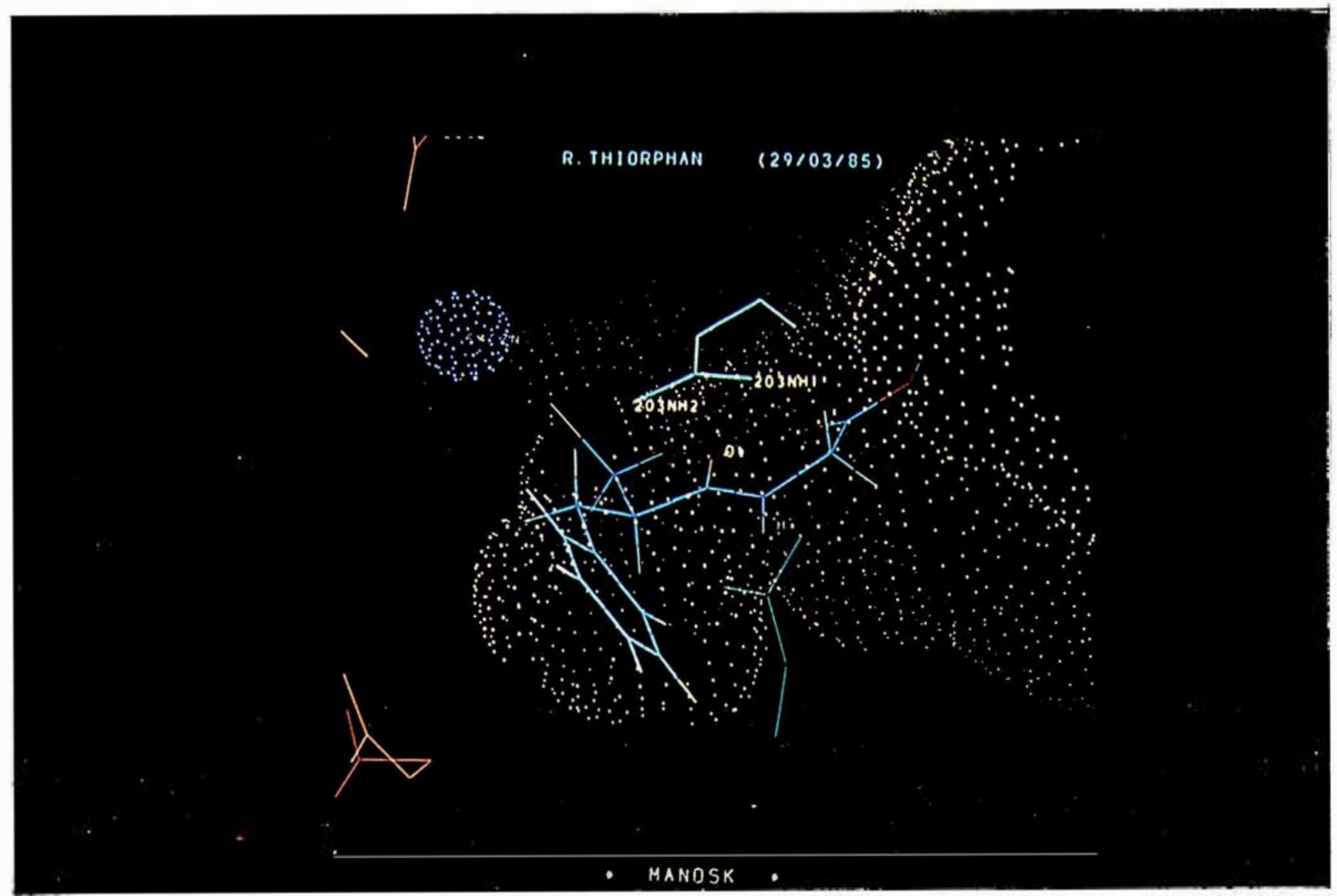

est probablement possible de préparer rationnellement une molécule organique de structure classique qui serait au $\mathrm{CCK}_{8}$, ce que la morphine est aux enképhalines. Un premier résultat prometteur va dans ce sens puisqu'un antagoniste non peptidique actif sur les récepteurs à CCK de la vésicule biliaire vient d'être isolé de milieu de culture d'aspergillus alliaceus [15].

\section{Récepteurs $\mu$ et $\delta$}

La mise au point d'agonistes et antagonistes opioïdes sélectifs a permis de montrer par autoradiographie la distribution distincte des récepteurs $\mu$ et $\delta$ dans le cerveau [16]. Cette distinction neuroanatomique pourrait rendre compte de l'implication préférentielle des sites $\mu$ dans la régulation supraspinale des influx nociceptifs [17]. De même, le fonctionnement des neurones dopaminergiques dans le striatum paraît être contrôlé par une stimulation sélective des récepteurs $\delta$ [ 18$]$. Les critères de sélectivité étant désormais satisfaits, il est évident que l'effort va porter sur une exploration précise par microscopie électronique de la localisation des différentes cibles des neuropeptides. Dans ce domaine, des outils sont à nouveau indispensables. La fixation irréversible par irradiation UV d'un peptide radiomarqué tel l'Azido DTLET qui porte une fonction photoactivable semble être une voie prometteuse [I9]

\section{Une nouvelle voie dans l'analgésie}

L'interruption de la transmission enképhalinergique est assurée par des peptidases qui clivent le peptide à différents niveaux en libérant des fragments inactifs :

\footnotetext{
dipeptidylaminopeptidase Tyr Gly $\left.\left.\right|^{\text {Gly }}\right|_{\text {enképhalinase, A }} ^{\text {Phe-Met (Leu) }}$

aminopeptidases
}

*Angiotensin I Converting Enzyme.

Toutes ces enzymes appartiennent au groupe des métallopeptidases. Les deux plus importantes sont l'enképhalinase et l'aminopeptidase $M$, toutes deux découvertes dans le rein mais également présentes dans le cerveau. La mise au point d'inhibiteurs de ces enzymes représentait une voie prometteuse pour étudier le rôle physiologique des enképhalines cérébrales et pour accéder à un nouveau type d'analgésie naturelle en augmentant la concentration des peptides opioïdes endogènes. Une des exigences à respecter était de synthétiser des composés bien évidemment incapables d'inhiber 


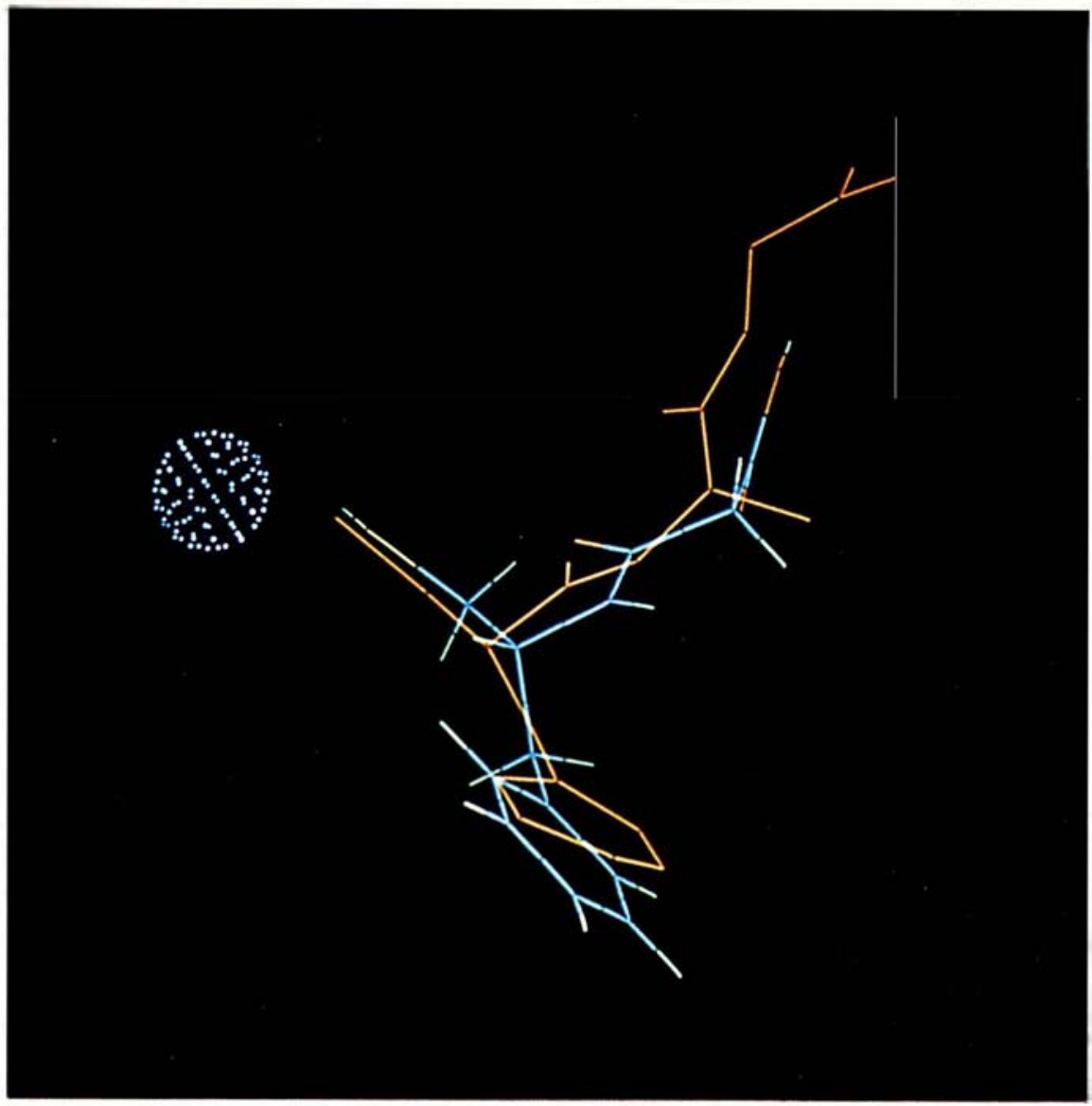

l'ACE puisque cette enzyme participe dans les vaisseaux au contrôle de la pression artérielle.

Pour préparer des inhibiteurs des enzymes de dégradation des enképhalines, nous nous sommes appuyés sur le modèle de site actif utilisé par Ondetti et Cuhsman lors de la mise au point de l'inhibiteur d'ACE, le captopril [20] et nous avons pris en compte les données cristallographiques d'une métallopeptidase bactérienne, la thermolysine [2I] qui, comme l'enképhalinase, est également capable d'hydrolyser la liaison $\mathrm{Gly}^{3}-\mathrm{Phe}^{4}$ des enképhalines.

Grâce à ces données cristallographiques, on sait à l'échelle atomique qu'un substrat se lie dans le site actif d'une métallopeptidase par coordination de l'atome d'oxygène de la liaison peptidique qui va être hydrolysée avec l'atome de métal du site catalytique (figure 4).
Figure 4. Visualisation sur écran graphique de l'inhibiteur thiorphan dans le site actif de la thermolysine, une enzyme bactérienne proche de l'enképhalinase (gauche) (Coll. 7. P. Mornon et E. Surcouf). Conformations du $R$-thiorphan et $d u$ R-rétro-thiorphan satisfaisant aux exigences d'interaction dans le site actif de la thermolysine (droite). L'analogie entre les deux structures permet de comprendre qu'en dépit de la rétro-inversion de la liaison amide, les deux molécules sont équiactives.

pour l'ACE. De manière à augmenter cette sélectivité nous avons préparé le rétro-thiorphan à partir de considérations sur la structure différentielle probable des sites actifs des deux métallopeptidases. La rétroinversion de la liaison amide dans $\mathrm{HS}-\mathrm{CH}_{2}-\mathrm{CH}\left(\mathrm{CH}_{2} \varphi\right) \mathrm{NHCO}-\mathrm{CH}_{2}-\mathrm{COOH}$, préserve donc l'activité envers l'enképhalinase mais supprime complètement la reconnaissance de l'ACE [23]. Par ailleurs, les deux inverses optiques $\mathrm{R}$ et $\mathrm{S}$ du thiorphan sont équiactifs alors que le rétro-thiorphan $R$ est 200 fois plus actif que l'isomère $\mathrm{S}$. La même dépendance stéréochimique est observée dans l'inhibition de la thermolysine par ces molécules.

Par ailleurs, la spécificité de ce type d'enzyme est assurée par l'existence de sous-sites adaptés à recevoir les chaînes latérales hydrophiles ou hydrophobes, chargées ou non du ou des substrats. Enfin un réseau de liaisons hydrogène s'établit entre l'enzyme et le substrat, ce qui impose la disposition spatiale de ce dernier dans la protéine.

La synthèse logique d'un inhibiteur de l'enképhalinase a consisté à explorer la spécificité des sous-sites puis à incorporer un groupe capable d'interagir très fortement avec l'atome de zinc de l'enzyme, dans une structure satisfaisant pleinement les exigences des sous-sites. C'est en partant de cette analyse qu'a été préparé le thiorphan : $\mathrm{HS}-\mathrm{CH}_{2}-\mathrm{CH}\left(\mathrm{CH}_{2} \varphi\right) \mathrm{CONH}-\mathrm{CH}_{2}-\mathrm{COOH}$. ${ }^{\text {er }}$ inhibiteur très puissant de l'enképhalinase $\left(\mathrm{K}_{\mathrm{I}} \sim 2 \mathrm{nM}\right)$ [22]. Le thiorphan est seulement 40 fois plus sélectif pour l'enképhalinase que
Ces résultats s'expliquent facilement en étudiant les possibilités d'adaptation conformationnelle au site actif de la thermolysine (et par analogie à celui de l'enképhalinase) des deux séries d'inhibiteurs (figure 4). L'utilisation des techniques de graphisme moléculaire qui permettent de visualiser sur écran les atomes constitutifs d'un site actif, puis d'y incorporer l'inhibiteur et de calculer les interactions entre les deux partenaires, sont évidemment d'un apport considérable dans la mise au point d'inhibiteurs puissants et sélectif $s$.

Comme attendu, thiorphan et rétro-thiorphan sont capables de protéger in vivo et in vitro les enképhalines de leur dégradation par l'enképhalinase et montrent de ce fait une action analgésique antagonisable par la naloxone [2 I, 23]. Néanmoins, les enképhalines sont 
également détruites par une activité aminopeptidasique et accessoirement par une dipeptidylaminopeptidase. Ces trois enzymes étant des métallopeptidases, il est théoriquement possible de prépaprer un composé capable de les inhiber toutes les trois. C'est ainsi qu'a été préparé le Kélatorphan [24] :

(R) $\mathrm{HONH}-\mathrm{CO}-\mathrm{CH}_{2}-\mathrm{CH}\left(\mathrm{CH}_{2} \varphi\right)$

$-\mathrm{CONH}-\mathrm{CH}\left(\mathrm{CH}_{3}\right)-\mathrm{COOH}$.

Ce composé est le premier inhibiteur complet du métabolisme des enképhalines. Il protège à $80-90 \%$ la dégradation des peptides opioides in vivo. Aussi actif que le thiorphan sur l'enképhalinase, il inhibe également la dipeptidylaminopeptidase $\left(\mathrm{K}_{1} \sim 2 \mathrm{nM}\right)$ et bloque sélectivement l'aminopeptidase M. Comme attendu, le Kélatorphan possède une action analgésique très supérieure au thiorphan [24]. En accord avec ses propriétés, ce composé est capable d'augmenter la libération basale d'enképhalines à la fois in vitro et in vivo et par conséquent de révéler les actions physiologiques soumises à un contrôle tonique par les peptides opioïdes [25]. Ainsi le Kélatorphan comme le DTLET, produit une augmentation de la libération d'enképhalines dans le striatum de rat [26]. Ce résultat très important laisse à penser que les enképhalines seraient impliquées (probablement au travers de la régulation des voies monoaminergiques) dans le contrôle de certains comportements et qu'un dérèglement de ce système pourrait être une des causes des dépressions endogènes, voire d'affections telles que la schizophrénie.

L'avenir thérapeutique des inhibiteurs potentiels du métabolisme des enképhalines repose entre autres sur la sélectivité des systèmes de dégradation. Une première réponse vient d'être apportée par la visualisation dans le cerveau de l'enképhalinase par autoradiographie à l'aide d'un inhibiteur tritié. L'enzyme possède une distribution discrète et une colocalisation satisfaisante avec les récepteurs opioïdes [27].

\section{Cibles du système enképhalinergique}

En dehors des récepteurs et des systèmes de dégradation, plusieurs cibles ont été étudiées par l'intermé- diaire de sondes spécifiques. D'abord une métalloenzyme dénommée enképhaline convertase [28], dont le rôle serait de libérer la Met-enképhaline par hydrolyse de la liaison Met-Arg du peptide TyrGly-Gly-Phe-Met-Arg. Celui-ci est obtenu par maturation du précurseur pro-enképhaline qui renferme cinq copies de Met-Enk encadrées de deux aminoacides basiques tels Arg-Lys ou Arg-Arg, qui servent de sites de clivage pour des enzymes probablement aspécifiques. Le rôle sélectif de l'enképhaline convertase dans la maturation ultime des enképhalines semble accréditée par la distribution de l'enzyme dans des régions riches en Met-enképhaline démontrée comme pour l'enképhalinase à l'aide d'un inhibiteur tritié [28].

Rien ne permet d'affirmer encore que la première étape de maturation de la pro-enképhaline nécessite une peptidase spécifique. Pourtant, le précurseur possède une structure tertiaire et par conséquent, probablement un seul site dibasique accessible à une peptidase qui pourrait être spécifique, compte tenu des contraintes structurales évidemment différentes pour chaque précurseur de neuropeptide. La séquence de la pro-enképhaline, étant connue grâce au génie génétique [29], on peut donc préparer des anticorps monoclonaux dirigés contre les différentes séquences $\ldots A_{n-2}-A_{n-1}-A_{n}$ précédant les séries Arg-Arg-Tyr-Gly ... et examiner l'inhibition de la première étape de maturation. Une autre approche plus élégante encore consiste à modifier par mutagénèse dirigée, un aminoacide potentiellement essentiel pour la reconnaissance par l'enzyme. De telles études sont actuellement en cours.

\section{Pharmacologie moléculaire du futur}

Les techniques d'hybridation in situ sont déjà utilisées couramment pour suivre, par exemple, l'influence d'un traitement aux neuroleptiques (halopéridol) ou d'électrochocs sur la synthèse d'enképhalines dans un tissu déterminé, en dosant l'ARN messager du précurseur et, en parallèle, la concentration du peptide lui-même et son taux de libération
[30]. Après l'application aux enzymes de dégradation (ce qui ne saurait tarder), puis plus tard aux récepteurs, on pourra alors étudier complètement le fonctionnement d'une voie neuronale. On conçoit l'importance de telles études dans l'étiologie et le traitement des maladies mentales ou dégénératives.

Il apparaît cependant que les plus grands progrès dans notre connaissance du système nerveux central, pourraient venir de l'application des techniques du "design " moléculaire à deux découvertes récentes. La première est la mise en évidence d'ARN messagers spécifiques aux cellules neuronales [3I]. Ces gènes expriment donc des protéines dont on ne connait pas les fonctions mais dont on peut reconstituer les séquences à partir de celles des messagers. Avec cette information, il est possible de "réprimer" spécifiquement l'expression d'une protéine en inhibant la traduction de son ARN messager, ou mieux, en dirigeant contre un déterminant de la protéine un anticorps obtenu à partir d'un peptide synthétique. Le choix des déterminants pourra s'effectuer dans un avenir proche en tenant compte des règles de repliement des protéines et donc d'apparition préférentielle à la surface des macromolécules de certaines séquences peptidiques.

La seconde application concerne la complémentarité entre les aminoacides correspondant à une séquence particulière sur l'ADN et ceux correspondant à la séquence autocomplémentaire. Ainsi, à un peptide comme l'ACTH correspond un peptide autocomplémentaire HTCA. Les deux molécules synthétiques interagissent fortement l'une avec l'autre d'où l'idée que le peptide HTCA pourrait faire partie du site actif d'une protéine réceptrice [32]. Cette hypothèse semble accréditée par le fait qu'un anticorps contre le peptide HTCA, se lie effectivement au récepteur de l'ACTH et inhibe la liaison de l'effecteur naturel. Si cette théorie était confirmée elle pourrait permettre de modéliser les interactions au sein d'un site récepteur sans connaître sa structure et faciliter par conséquent la mise au point d'agonistes et d'antagonistes. Par ailleurs, les anticorps agissant 
contre le peptide " autocomplémentaire " pourraient permettre d'isoler plus facilement les récepteurs.

Enfin, la constatation d'un constant décalage topologique en autoradiographie entre la distribution des peptides et leur site de liaison [33], conduit à la notion de transmission non synaptique à courte distance qui pourrait être étayée par une localisation non neuronale de certaines enzymes du métabolisme des peptides (résultats en cours de publication). De nouvelles méthodes de visualisation en microscopie électronique sont nécessaires pour confirmer ou infirmer ces hypothèses qui pourraient expliquer par exemple l'affinité très forte des récepteurs aux neuropeptides par rapport aux effecteurs classiques (acétylcholine, monoamines....) et surtout conforteraient le rôle de plus en plus important accordé aux cellules gliales [34] dans le fonctionnement du système nerveux central

\section{Summary}

Progress in molecular biology has allowed to characterize new neuronal circuits, whose natural effectors are peptides, such as enkephalins (endogenous morphins) or brain cholecystokinin CCK 8. These molecules are synthetized as large precursors. They bind to several classes of receptors. They are degraded by specific enzyme systems. The analysis of the fonction of a neuropeptidergic system requires chemical tools with the ability to interfere selectively with regulatory targets. Such compounds are obtained by taking into account the conformational adaptability of the endogenous effector which allows its binding to several sites. From nuclear magnetic resonance data, models of speci- fic interaction with the opioid receptors $\mu$ and $\delta$, and with the sites for CCK 8 have been defined; they allow to synthetize highly specific agonists and antagonists. Likewise, Kelatorphan, an inhibitor of enkephalin degradation enzymes, has been obtained from cristallographic data on thermolysin, a bacterial metallopeptidase. The techniques of molecular genetics now allow to follow both the synthesis of a neuropeptide (by measuring its precursor $m$ RNA) and its release into the neuronal space (by immunocytochemistry). These methods should also permit to define the role of the peptides selectively expressed in neurones by inhibiting their expression.

\section{TIRÉS A PART}

B. Roques : U 266 Inserm, UA 498 Cnrs, 4, avenue de l'Observatoire, 75270 Paris Cedex o6. 